\title{
Psicooncología
}

ISSN: 1696-7240

\section{Efecto de una intervención de enfermería en mujeres con cáncer de mama en el postoperatorio}

\author{
Mónica Liliana Mesa ${ }^{1}$; Gloria Mabel Carrillo,; Olga Janeth Gómez ${ }^{3}$; Lorena Chaparro \\ Díaz ${ }^{4}$ Sonia Patricia Carreño ${ }^{5}$
}

Recibido: 19 de marzo de 2019 / Aceptado: 5 de septiembre de 2019

Resumen: Objetivos: Determinar el efecto de la intervención educativa de enfermería en mujeres con cáncer de mama durante el postoperatorio para fortalecer la competencia de cuidado en el hogar. Método: Se trata de un abordaje cuasi experimental, que conto con un total de 88 mujeres. El grupo de intervención estuvo constituido por 58 participantes y el control por 30. Las mujeres en los dos grupos recibieron un cuestionario que evalúo su competencia para el cuidado en el hogar al ingreso hospitalario. Una intervención de enfermería se aplicó desde el ingreso hasta cuatro semanas después de la cirugía con el grupo de intervención mientras que el control continuo recibiendo la intervención convencional. Los dos grupos aplicaron nuevamente el cuestionario de competencia para el cuidado en el hogar 4 semanas después del egreso. Resultados: En el grupo intervenido, la diferencia entre los puntajes de las medias totales de la competencia para el cuidado en el hogar aumentó significativamente comparado con el control, y en las dimensiones conocimiento, unicidad y bienestar.

Conclusiones: La intervención de enfermería estructurada es una estrategia viable que aumenta la competencia para cuidar en el hogar, en mujeres con cáncer de mama sometidos a cirugía.

Palabras clave: Alta del paciente, mastectomía, educación, neoplasias de la mama, enfermería oncológica, proyectos de investigación.

\section{[en] Effect of a nursing intervention in women with postoperative breast cancer}

\footnotetext{
Abstract: Objectives: To determine the effect of the nursing educational intervention in women with breast cancer during the postoperative period to strengthen the competence of home care. Method: This is a quasi-experimental approach, which involved a total of 88 women. The intervention group consisted of 58 participants and control by 30 . The women in the two groups received a questionnaire

1 Mónica Liliana Mesa. Enfermera. Especialista en Enfermería. Magister en Enfermería. Enfermera Grupo Área Enfermería. Instituto Nacional de Cancerología. Bogotá, Colombia.

E-mail: mlpedreros@cancer.gov.co

2 Gloria Mabel Carrillo. Facultad de Enfermería. Universidad Nacional de Colombia. Bogotá, Colombia. E-mail: gmcarrillog@unal.edu.co

3 Olga Janeth Gómez. Facultad de Enfermería. Universidad Nacional de Colombia. Bogotá, Colombia. E-mail: ojgomezr@unal.edu.co

4 Lorena Chaparro Díaz. Facultad de Enfermería. Universidad Nacional de Colombia. Bogotá, Colombia. E-mail: olchaparrod@unal.edu.co

5 Sonia Patricia Carreño. Facultad de Enfermería. Universidad Nacional de Colombia. Bogotá, Colombia. E-mail: spcarrenom@unal.edu.co

* Dirección de correspondencia: Gloria Mabel Carrillo. Universidad Nacional de Colombia. Sede Bogotá-Departamento de Enfermería. Cra 30 \# 45-03. Edificio 228 Facultad de Enfermería, Of 301. Bogotá D.C.,Colombia. E-mail: gmcarrillog@unal.edu.co
} 
that assessed their competence for home care at hospital admission. A nursing intervention was applied from admission until four weeks after surgery with the intervention group while continuous control receiving conventional intervention. The two groups applied again the competence questionnaire for home care 4 weeks after discharge. Results: In the group operated, the difference between the scores of the total means of competition for home care increased significantly compared to the control, and in the knowledge, uniqueness and well-being dimensions. Conclusions: Structured nursing intervention is a viable strategy that increases the competence to care at home, in women with breast cancer undergoing surgery.

Keywords: Patient discharge, mastectomy, education, breast neoplasms, oncology nursing, research design.

Sumario. 1. Introducción 2. Método 3. Resultados 4. Discusión 5. Conclusiones 6. Referencias bibliográfica

Cómo citar: Mesa ML, Carrillo GM, Gómez OJ, Chaparro Díaz L, Carreño SP. Efecto de una intervención de enfermería en mujeres con cáncer de mama en el postoperatorio. Psicooncología 2019; 16: 273-286. doi: 10.5209/psic.65591

\section{Introducción}

Existe un crecimiento significativo en la incidencia de cáncer de mama en la población femenina contemporánea. Una de cada 18 mujeres desarrolla cáncer de mama antes de los ochenta años ${ }^{(1)}$.

El cáncer de mama es la primera causa de enfermedad y muerte por cáncer entre las mujeres colombianas ${ }^{(2)}$. El número de casos reportados por GLOBOCAN 2018 corresponden a $13.380(14,73 \%)$, con una mortalidad de 3.702 casos $(8,89 \%)$, y una prevalencia de 39.330 casos para los últimos cinco años ${ }^{(3)}$.

La mastectomía como uno de los tratamientos centrales en la atención de pacientes con cáncer de mama implica un proceso de adaptación e impacto en la calidad de vida, en especial, en las esferas físicas y psicológicas ${ }^{(1)}$. El procedimiento quirúrgico se percibe como un evento traumático ${ }^{(4,5)}$ que puede resultar en un estado de estrés psicológico con insatisfacción y pobre bienestar por variables como los cambios en la imagen corporal, en la feminidad y los procesos de reconstrucción ${ }^{(6,7)}$. De otra parte, la cirugía a menudo se realiza poco después del diagnóstico cuando la paciente puede estar en un estado de angustia emocional lo que resulta en sobrecarga de información ${ }^{(8)}$.

La participación de la paciente en el cuidado de la salud es esencial para aumentar la seguridad y bienestar, la satisfacción, la calidad de vida y disminuir los costos del tratamiento ${ }^{(9)}$. En el caso de las mujeres con cáncer de mama sometidas a intervenciones quirúrgicas las estancias hospitalarias tienden a ser más cortas ${ }^{(10)}$, sin embargo las demandas de cuidado son altas, requiriendo la generación de intervenciones que faciliten mayor conocimiento de la situación en vez de tanta información, y mejor interacción con los profesionales del área de la salud ${ }^{(11)}$.

Se requiere preparación desde los centros de atención en aspectos instrumentales como el cuidado de las heridas, manejo de dolor, monitorización de complicaciones para contribuir a minimizar los síntomas y molestias postquirúrgicas, a una mayor optimización de los servicios y disminuir las readmisiones ${ }^{(12)}$.

Las intervenciones en pacientes con cáncer de mama sometidas a cirugía en alguna fase del proceso perioperatorio han demostrado efectos en disminución de 
la ansiedad y angustia, el automonitoreo de los sistemas de drenaje continuo ${ }^{(13)}$, los conocimientos sobre el diagnóstico y tratamiento, manejo de linfedema ${ }^{(14)}$, efectos secundarios del tratamiento $^{(15)}$, bienestar y felicidad ${ }^{(16)}$. A pesar de lo descrito, la evidencia es limitada sobre los potenciales beneficios que proveen esfuerzos de estas características en el proceso perioperatorio de pacientes con cáncer de mama, los resultados reflejan heterogeneidad de intervenciones.

De otra parte, el rol de enfermería es fundamental en estas intervenciones, e implican el diseño de estrategias para estas pacientes, teniendo en cuenta sus características, el tiempo, los contenidos educativos y de conocimiento, el propósito y el tipo de seguimiento postoperatorio a desarrollar ${ }^{(17)}$. Se constituye este tipo de intervención como un enfoque innovador de la atención en oncología en el contexto colombiano que involucra a todo el equipo de salud, resaltando el rol de enfermería.

Por lo tanto, el propósito del estudio fue evaluar el efecto de una intervención educativa de enfermería en mujeres con cáncer de mama durante el postoperatorio para fortalecer la competencia de cuidado en el hogar en las dimensiones conocimiento, unicidad, instrumental, disfrutar, anticipación y relación e interacción social.

\section{Método}

Estudio cuasi experimental con un diseño de pruebas pre y pos con grupo de intervención (GI) y grupo control (GC) y medición de la competencia para el cuidado en el hogar de los pacientes antes y después de la intervención.

El estudio es un resultado secundario del protocolo aprobado por el Comité de Ética e Investigación del Instituto Nacional de Cancerología (Bogotá-Colombia). Este hospital es un centro de referencia en oncología en Colombia, con unidades especializadas para la atención quirúrgica de pacientes con cáncer de mama. Se condujo desde noviembre de 2016 a mayo de 2018. El consentimiento informado fue obtenido desde la captación de las mujeres a partir de una explicación detallada del propósito del estudio, los procesos de recolección y análisis de datos, la confidencialidad y la posibilidad de declinar su participación sin consecuencia alguna.

Todas las participantes fueron reclutadas a partir de los siguientes criterios de inclusión: a. mayores de 18 años, b. Con diagnóstico de cáncer de mama. c. En postoperatorio de cirugía oncológica con intención curativa en los servicios de quirúrgicas y d. Estables hemodinamicamente.

Se contó con una lista de programación de cirugía cada semana, para la ubicación de las pacientes en cada uno de los grupos. Todas los pacientes fueron monitorizados por profesionales de enfermería en varios momentos: El día del reclutamiento, antes de la cirugía, durante el postoperatorio en los servicios de recuperación y hospitalización, y entre la primera y cuarta semana después del egreso hospitalario. Cada encuentro duro entre 20 a 60 minutos. Figura 1.

Los cuestionarios fueron administrados por los auxiliares de investigación después del reclutamiento, con excepción de CUIDAR que fue auto-diligenciado por las pacientes. 
Figura 1. Diagrama de flujo de los procedimientos para la inclusión, asignación, seguimiento y análisis de la muestra del estudio

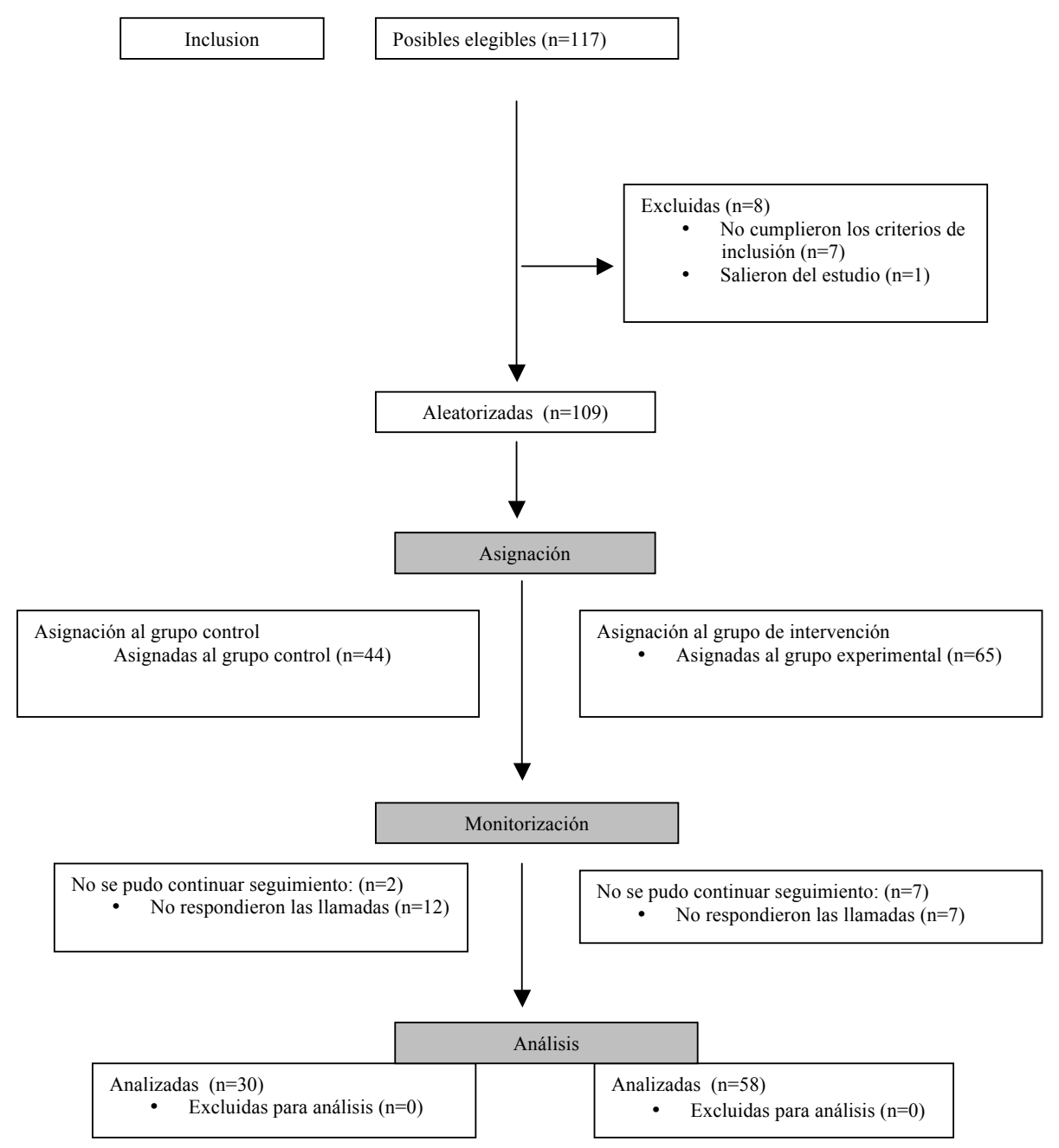

Los instrumentos utilizados fueron:

1. Caracterización del paciente: Ítems que exploran aspectos del paciente respecto a edad, género, estado civil, ocupación, estrato socioeconómico, diagnóstico médico e intervención quirúrgica a realizar.

2. CUIDAR: Competencia para el cuidado en el hogar versión paciente ${ }^{(18)}$. Se define teóricamente como la capacidad, habilidad y preparación que tiene la persona para ejercer la ejercer la labor de cuidar en el hogar. El instrumento comprende 6 categorías que se ordenan bajo el acróstico CUIDAR (por su fácil recordación): Conocimiento, Unicidad (condiciones personales), Instrumental, Disfrutar (bienestar), Anticipación, y Relación social e interacción. Consta de 
2 versiones: una para el paciente y otra para el cuidador familiar, tiene 20 ítems que se miden a través de escala tipo Likert con puntuaciones que van de 0 a 3; 0 es nunca, 1 pocas veces, 2 con frecuencia y 3 casi siempre o siempre. Cuenta con estratificación en rangos alto, medio y bajo con puntajes más altos que indican mayor grado de competencia. Respecto a sus propiedades psicométricas tiene un índice de validez de contenido global de 0,97 . El método de extracción y el análisis de componentes principales con los seis factores propuestos indican una varianza total explicada del $72,94 \%$. La confiabilidad mediante el alfa de Cronbach fue de 0,928 para el instrumento en su totalidad, con valores entre 0,926 y 0,930 en cada una de las dimensiones ${ }^{(18)}$.

La intervención de enfermería consistió en una estrategia coordinada y ejecutada por personal de enfermería que valora al paciente desde el ingreso a la institución, e incluye el encuentro en cinco sesiones, con abordaje de temas como manejo de medicamentos parenterales, dispositivos invasivos, entrega de material educativo de acuerdo a la prescripción médica de egreso, instrucción e información específica cara a cara en el postoperatorio, taller demostrativo sobre manejo de dispositivos invasivos, y llamada telefónica una vez a la semana durante 4 semanas con una guía previamente establecida de tópicos a abordar. (Tabla 1)

Tabla 1. Intervención de enfermería para mujeres con cáncer de mama en el postoperatorio

\begin{tabular}{|c|c|c|c|}
\hline Sesiones & CONTENIDOS & DURACIÓN & OBSERVACIONES \\
\hline I & $\begin{array}{l}\text { Conocimiento enfermedad y su significa- } \\
\text { do, tratamiento quirúrgico. } \\
\text { Información sobre dieta y alimentación, } \\
\text { ejercicio, actividades de la vida diaria. } \\
\text { Búsqueda y utilización de redes de apoyo }\end{array}$ & 1 hora & $\begin{array}{l}\text { Durante la estancia } \\
\text { hospitalaria. } \\
\text { Presencial }\end{array}$ \\
\hline 2 & $\begin{array}{l}\text { Taller demostrativo: manejo de sistemas } \\
\text { de drenaje, anticoagulantes, uso de medias } \\
\text { antie-embólicas, medicamentos. } \\
\text { Identificación de signos de alarma } \\
\text { Entrega de material educativo. }\end{array}$ & 1 hora & $\begin{array}{l}\text { Durante la estancia } \\
\text { hospitalaria. } \\
\text { Presencial }\end{array}$ \\
\hline \multirow[t]{2}{*}{3} & $\begin{array}{l}\text { Refuerzo manejo de sistema de drenajes, } \\
\text { anticoagulantes y medicamentos. } \\
\text { Identificación de signos de alarma }\end{array}$ & \multirow[t]{2}{*}{20 minutos } & \multirow[t]{2}{*}{$\begin{array}{l}\text { Vía telefónica } \\
5 \text { a } 7 \text { días después del } \\
\text { egreso }\end{array}$} \\
\hline & $\begin{array}{l}\text { Re incorporación a las actividades de la vida } \\
\text { diaria y modificación de estilos de vida. }\end{array}$ & & \\
\hline 4 & $\begin{array}{l}\text { Identificación de signos de alarma. } \\
\text { Pensando en un plan de vida. } \\
\text { Manejando necesidades de cuidado en } \\
\text { casa } \\
\text { Utilización de redes de apoyo }\end{array}$ & 20 minutos & $\begin{array}{l}\text { Egreso-. Vía } \\
\text { telefónica } \\
12 \text { a } 14 \text { días después } \\
\text { del egreso }\end{array}$ \\
\hline 5 & $\begin{array}{l}\text { Plan de vida. } \\
\text { Reintegro a la vida laboral } \\
\text { Ajustes a los estilos de vida. }\end{array}$ & 20 minutos & $\begin{array}{l}\text { Egreso- vía telefónica } \\
23 \text { a } 27 \text { días después } \\
\text { del egreso. }\end{array}$ \\
\hline
\end{tabular}

Fuente: Construcción propia autores. 2017 
La intervención convencional en la institución para este tipo de pacientes consiste en entregar material educativo de acuerdo a la prescripción médica y taller demostrativo para el manejo de los drenajes. Las sesiones son individuales para cada paciente, siendo opcional la participación de la familia.

Cuatro semanas después del egreso los participantes de los dos grupos diligenciaron nuevamente el instrumento CUIDAR.

En el análisis de datos, se utilizó el paquete SPSS versión 22.0. Las variables continuas fueron expresadas en la media y desviación estándar, las variables categóricas en porcentajes. Para la comparación de la variable CUIDAR se proyectó usar ANOVA de medidas independientes y $\mathrm{t}$-student para muestras repetidas, previa verificación del supuesto de normalidad, mediante la prueba de KolmogorovSmirnov. Se consideraron estadísticamente significativos los valores de $\mathrm{p}<0,05$.

Dentro de las consideraciones éticas se tuvo en cuenta las pautas para la investigación biomédica preparadas por el Consejo de Organizaciones Internacionales de las Ciencias Médicas (CIOMS) ${ }^{(19)}$ y los parámetros establecidos en la Resolución 8430 de 1993 expedida por el Ministerio de Salud de Colombia ${ }^{(20)}$. Se contemplaron aspectos relacionados con el consentimiento informado, la participación voluntaria y el manejo confidencial de la información.

\section{Resultados}

En la caracterización se evidencia la homogeneidad en la composición del GC y el GI, sin cambios estadísticamente significativos en las variables: edad, nivel de dependencia del paciente, nivel de escolaridad, estado mental, ocupación y estrato socioeconómico (Tabla 2).

En la competencia para el cuidado de las pacientes, antes de la intervención, no existen diferencias apreciables entre el GI y el GC. En cada uno de los grupos, las diferencias existentes en las medias de los índices de competencia, no son estadísticamente significativas.

Aunque posterior a la intervención se obtienen cambios a nivel descriptivo en la competencia para el cuidado en el hogar en los dos grupos son contundentes los mayores niveles obtenidos en el GI frente al GC. En el GI se identifican cambios estadísticamente significativos en la competencia para el cuidado en el hogar y en las dimensiones conocimiento, unicidad y disfrutar; en contraste en el GC la competencia global y en los componentes de conocimiento y unicidad y disfrutar aumenta sin significancia estadística y en las dimensiones instrumental, anticipación y relación se disminuyen los valores (Tabla 3 ).

De otra parte, hay diferencias estadísticamente significativas entre el GI y el GC en la competencia para el cuidado en el hogar, y en todas las dimensiones con excepción de la relación e interacción social (Tabla 4).

En cuanto al tamaño del efecto que corresponde a la diferencia entre el grupo control y el de intervención; la competencia para el cuidado en el hogar presenta un valor de diferencia de medias estandarizada ( $\mathrm{d}$ de Cohen) mayor a 0,6 lo que indica un tamaño de efecto mediano de la intervención. 
Tabla 2. Características sociodemográficas de la muestra

\begin{tabular}{|c|c|c|c|c|}
\hline Variables & & $\begin{array}{l}\text { Grupo } \\
\text { intervención } \\
n=58\end{array}$ & Grupo control $=30$ & $\mathrm{P}$ valor \\
\hline Edad & & $54,3+/-12,4$ & $57,4+/-10,4$ & $0.465^{*}$ \\
\hline \multirow[t]{2}{*}{ Dependencia(\%) } & $\begin{array}{l}\text { Baja } \\
\text { dependencia }\end{array}$ & 98,3 & 100 & $0,469 *$ \\
\hline & $\begin{array}{l}\text { Mediana } \\
\text { dependencia }\end{array}$ & 1,7 & & \\
\hline \multirow[t]{2}{*}{ Estado mental (\%) } & Intacto & 98,3 & 100 & $0,469^{*}$ \\
\hline & alteración leve & 1,7 & & \\
\hline \multirow{4}{*}{$\begin{array}{l}\text { Grado de escolaridad } \\
(\%)\end{array}$} & Bajo & 72,4 & 76,7 & $0,522 *$ \\
\hline & Medio & 5,2 & 13,3 & \\
\hline & Alto & 19,0 & 6,7 & \\
\hline & Ninguno & 3,4 & 3,3 & \\
\hline \multirow[t]{5}{*}{ Estado civil (\%) } & Soltera & 19,0 & 30,0 & $0,522 *$ \\
\hline & Casada & 32,8 & 36,7 & \\
\hline & Separada & 10,3 & 13,3 & \\
\hline & Viuda & 13,8 & 6,7 & \\
\hline & Unión libre & 24,1 & 13,3 & \\
\hline \multirow[t]{6}{*}{ Ocupación (\%) } & Hogar & 62,0 & 70,0 & $0,485^{*}$ \\
\hline & Empleado & 13,8 & 3,3 & \\
\hline & $\begin{array}{l}\text { Trabajo } \\
\text { Independiente }\end{array}$ & 17,2 & 23,3 & \\
\hline & Pensionado & 1,7 & 3,3 & \\
\hline & Oficios varios & 1,7 & 0 & \\
\hline & Estudiante & 3,4 & 0 & \\
\hline \multirow{4}{*}{$\begin{array}{l}\text { Estrato } \\
\text { socioeconómico }(\%)\end{array}$} & 1 & 34,5 & 23,3 & $0,602 *$ \\
\hline & 2 & 39,7 & 50,0 & \\
\hline & 3 & 24,1 & 16,7 & \\
\hline & 4 & 1,7 & & \\
\hline
\end{tabular}

La variable continua edad fue expresada como media +/-, desviación típica, las variables categóricas fueron expresadas en porcentajes.* Independent T-test ; **Chi-square test. 
Tabla 3. Competencia para el cuidado en el hogar y sus dimensiones, antes y después de la intervención. Diferencia de medias por cada grupo

\begin{tabular}{|c|c|c|c|c|}
\hline \multirow[t]{2}{*}{ GRUPO } & \multirow[t]{2}{*}{ VARIABLE } & $\begin{array}{l}\text { Antes de la } \\
\text { intervención }\end{array}$ & $\begin{array}{l}\text { Después de la } \\
\text { intervención }\end{array}$ & \multirow[t]{2}{*}{ P valor* } \\
\hline & & Media & Media & \\
\hline \multirow{7}{*}{ INTERVENCIÓN } & $\begin{array}{l}\text { Competencia para el } \\
\text { cuidado }\end{array}$ & 51,84 & 57,5 & $0,00 * *$ \\
\hline & Conocimiento & 5,46 & 8,3 & $0,000 * *$ \\
\hline & Unicidad & 10,6 & 11,5 & $0,000 * *$ \\
\hline & Instrumental & 8,75 & 8,9 & 0,16 \\
\hline & Disfrutar & 9,89 & 11,1 & $0,02 * *$ \\
\hline & Anticipación & 5,56 & 5,8 & 0,41 \\
\hline & Relación e interacción & 11,55 & 11,6 & 0,15 \\
\hline \multirow{7}{*}{ CONTROL } & $\begin{array}{l}\text { Competencia para el } \\
\text { cuidado }\end{array}$ & 52,00 & 53,9 & 0,35 \\
\hline & Conocimiento & 5,16 & 7,6 & 0,22 \\
\hline & Unicidad & 10,4 & 10,7 & 0,45 \\
\hline & Instrumental & 8,8 & 8,3 & 0,12 \\
\hline & Disfrutar & 9,8 & 9,9 & 0,72 \\
\hline & Anticipación & 5,8 & 5,4 & 0,37 \\
\hline & Relación e interacción & 11,9 & 11,8 & 0,25 \\
\hline
\end{tabular}

$* \mathrm{t}$ student test para medidas repetidas $* * \mathrm{p}<0,05$

Las dimensiones instrumental y anticipación reportan un tamaño de efecto grande (superiores a 0,8 ). Sin embargo, los componentes de conocimiento, unicidad, disfrutar y relación e interacción social indican un tamaño de efecto pequeño (menores a 0,5 ) (véase Tabla 5). 
Tabla 4. Competencia para el cuidado en el hogar y sus dimensiones, antes y después de la intervención en la muestra. Diferencia de medias entre grupos

\begin{tabular}{|c|c|c|c|c|c|c|}
\hline & \multicolumn{3}{|c|}{ Antes de la intervención } & \multicolumn{3}{|c|}{ Después de la intervención } \\
\hline & Media & Desv. Típica & $\mathrm{p}$ valor* & Media & Desv. Típica & $\mathrm{p}$ valor* \\
\hline \multicolumn{7}{|c|}{ Competencia para el cuidado en el hogar } \\
\hline GI & 51,84 & 6,3 & \multirow[t]{2}{*}{0,91} & 57,5 & 2,65 & \multirow[t]{2}{*}{$0,00 * *$} \\
\hline GC & 52 & 5,8 & & 53,9 & 6,3 & \\
\hline \multicolumn{7}{|c|}{ Conocimiento } \\
\hline GI & 5,46 & 2,9 & \multirow[t]{2}{*}{0,67} & 8,3 & 0,9 & \multirow[t]{2}{*}{$0,01 * *$} \\
\hline GC & 5,16 & 3,5 & & 7,6 & 1,8 & \\
\hline \multicolumn{7}{|c|}{ Unicidad } \\
\hline GI & 10,6 & 1,8 & \multirow[t]{2}{*}{0,75} & 11,5 & 0,8 & \multirow[t]{2}{*}{$0,006 * *$} \\
\hline $\mathrm{GC}$ & 10,4 & 2,1 & & 10,7 & 1,6 & \\
\hline \multicolumn{7}{|c|}{ Instrumental } \\
\hline GI & 8,75 & 0,8 & \multirow[t]{2}{*}{0,8} & 8,9 & 0,2 & \multirow[t]{2}{*}{$0,001 * *$} \\
\hline $\mathrm{GC}$ & 8,8 & 0,5 & & 8,3 & 1,2 & \\
\hline \multicolumn{7}{|c|}{ Disfrutar } \\
\hline GI & 9,89 & 2,6 & \multirow[t]{2}{*}{0,91} & 11,1 & 1,5 & \multirow[t]{2}{*}{$0,02 * *$} \\
\hline $\mathrm{GC}$ & 9,8 & 2,5 & & 9,9 & 2,1 & \\
\hline \multicolumn{7}{|c|}{ Anticipación } \\
\hline GI & 5,56 & 0,9 & \multirow[t]{2}{*}{0,21} & 5,8 & 0,4 & \multirow[t]{2}{*}{$0,006 * *$} \\
\hline $\mathrm{GC}$ & 5,8 & 0,6 & & 5,4 & 1,2 & \\
\hline \multicolumn{7}{|c|}{ Relación e interacción } \\
\hline GI & 11,55 & 1,3 & \multirow[t]{2}{*}{0,13} & 11,6 & 1,08 & \multirow[t]{2}{*}{0,35} \\
\hline GC & 11,9 & 0,2 & & 11,8 & 0,5 & \\
\hline
\end{tabular}

GI: Grupo de intervención, GC: Grupo de Control. *ANOVA Test para medidas independientes. $* * p<0,05$.

Tabla 5. Tamaño de efecto d de Cohen. Competencia para el cuidado en el hogar

\begin{tabular}{|l|l|}
\hline Variable & Diferencia de medias estandarizada (d cohen) \\
\hline Competencia para el cuidado en el hogar & 0,61 \\
\hline Conocimiento & 0,12 \\
\hline Unicidad & 0,30 \\
\hline Instrumental & 0,96 \\
\hline Disfrutar & 0,46 \\
\hline Anticipación & 0,83 \\
\hline Relación e interacción social & 0,16 \\
\hline
\end{tabular}




\section{Discusión}

El estudio reporta la eficacia de una intervención de enfermería que incluye instrucción e información brindada cara a cara desde el ingreso a la institución, talleres y seguimiento telefónico hasta un mes después del alta, para aumentar la competencia de cuidado en el hogar del GI respecto al GC. Esta competencia es una variable central de proceso y resultado proximal en la atención de salud, que involucra tanto aspectos procedimentales e informacionales respecto al manejo del cáncer y la intervención quirúrgica, como en aquellas dimensiones que son determinantes para la recuperación: condiciones personales de la paciente, la percepción de bienestar, y la relación e interacción social, así como la capacidad de toma de decisiones para anticiparse en el abordaje de aspectos concernientes a la propia salud. De esta forma, se busca consolidar una estrategia integral, que sea de fácil aplicación y factible de replicar en los servicios de salud del contexto local.

La intervención de enfermería bajo un esquema documentado y con un sistema de registro diferenciando las necesidades del paciente y usando una valoración estándar aumenta de forma significativa la competencia para el cuidador en el hogar, hallazgos que concuerdan con dos investigaciones adelantadas que mantienen el mismo esbozo en el contexto colombiano con pacientes crónicos ${ }^{(21,22)}$.

El perfil de las pacientes en cuanto a la edad, tipo de cirugía y estadio reflejan la problemática de esta neoplasia en el contexto colombiano ${ }^{(23)}$. Las condiciones socioeconómicas y el nivel educativo de las participantes, se vislumbran como variables a ser analizadas por el equipo de salud en los programas de seguimiento, ya que son factores que las hacen más vulnerables a complicaciones relacionadas con el manejo de su condición de salud.

El tipo de intervención consolidada refleja la necesidad de contar con un equipo de enfermeras formalmente constituido que pueda proveer soporte permanente y feedback a los participantes con instrucciones cortas y sencillas, orientaciones sobre la transición desde el hospital y el hogar, así como utilización de redes de apoyo disponibles, al igual que lo referido en otro estudio ${ }^{(24)}$.

La competencia para el cuidado en el hogar, desde la dimensión de conocimiento reporta cambios significativos tanto en el GI como en el GC, siendo más alto el valor obtenido en el GI, la información brindada respecto a la enfermedad, las indicaciones de la terapéutica y las actividades a realizar en la cotidianidad, es efectiva tanto en la intervención convencional que se da en la institución como en la intervención propuesta. Una revisión de literatura realizada en $2018^{(25)}$ reporta hallazgos similares en cuanto al efecto que trae la información dada a las pacientes con cáncer de mama en el bienestar físico, el psicológico, el social y el espiritual. Esta información comprende aspectos tales como el auto-manejo, el afrontamiento, soporte psico-educativo, estilos de vida, imagen corporal, y salud mental ${ }^{(26)}$.

Se identifica, además, la importancia de realizar talleres demostrativos con la paciente a fin de potenciar habilidades y destrezas en manejo de dispositivos, alimentación y medicamentos en casa, y brindar instrucción personalizada sobre la planeación ante la ocurrencia de eventos inesperados, identificación de riesgos y uso racional de servicios de salud. Evidencia que coincide con otro estudio en el que estrategias educativas con simulación y seguimiento telefónico favorece la prevención de complicaciones en pacientes con cáncer de $\operatorname{mama}^{(13)}$. 
En las dimensiones de unicidad y bienestar, es necesario entender lo que se significa el tratamiento para estas pacientes, en muchos casos es aterrador y estresante, con alta carga de incertidumbre, ansiedad y preocupaciones que se reportan durante el seguimiento. Las decisiones que se toman pueden cambiar la vida por completo, situación que desencadena mayor ansiedad e inseguridad sobre el futuro ${ }^{(27)}$. En consecuencia, indagar en aspectos básicos de la vida cotidiana, así como la satisfacción personal relacionada con la calidad de vida percibida, son factores fundamentales a ser explorados como parte de la intervención integral del equipo de salud. Es de señalar, que en nuestro estudio estas dimensiones reportaron cambios significativos en el grupo intervenido, hallazgo que coincide con otros en el que el apoyo de enfermeras "navegadoras" que coordinaban la atención y acceso a la información, benefició a pacientes con cáncer que presentaban síntomas emocionales durante el diagnóstico y tratamiento con impacto sobre la calidad de vida y disminución de problemas psicosociales ${ }^{(28,29)}$.

El componente de relación e interacción, se constituye como un factor protector y posiblemente amortiguador en el manejo del postoperatorio de estas pacientes ya que se reportan altos niveles previo a la intervención en los dos grupos, es decir, cuentan con redes de apoyo primarias y secundarias como factores que favorecen el fortalecimiento de la labor de cuidado. Es probable que las participantes estén en una etapa del proceso en la que se tiende a reunir la mayor parte de la familia para apoyarla, por ello se requiere mantener dichas redes, que se pueden fracturar o debilitar en etapas más avanzadas en las que aparecen los efectos secundarios de tratamientos adyuvantes, la dependencia y hay mayores demandas de cuidado.

El estudio presenta varias limitaciones. Los resultados son parciales debido a los desafíos que representó la inclusión de una población específica de pacientes con diagnóstico de cáncer de mama en tratamiento de cirugía, así como la dificultad para realizar aleatorización y asignación igual para los dos grupos, la atrición en el seguimiento es una situación de especial atención ya que muchas pacientes viven en otras ciudades diferentes a Bogotá, situación que limita mantener una comunicación permanente con los pacientes por que se remiten para continuar en control con las instituciones primarias de atención. Se requiere consolidar estrategias de seguimiento para este tipo de población con uso de otros medios de comunicación además del teléfono, y establecer canales de información con los centros de atención primaria. Ahora bien, es necesario determinar qué otras variables influyen en las dimensiones que reportan tamaños de efecto pequeños en la competencia para el cuidado en el hogar de estas mujeres. Futuras investigaciones requieren profundizar en el comportamiento de la competencia para el cuidado en el hogar a mediano y largo plazo, es fundamental incluir otras variables de resultado como la calidad de vida percibida y la carga del síntoma; y explorar la intervención en tratamientos adyuvantes como quimioterapia y radioterapia.

\section{Conclusiones}

La intervención educativa de enfermería en pacientes con cáncer de mama sometidos a cirugía, aumenta la competencia de cuidado en el hogar, en las dimensiones conocimiento, unicidad, instrumental, disfrutar y anticipación.

El mayor efecto de la intervención se obtiene sobre las dimensiones instrumental y anticipación. 
Los resultados del estudio orientan al equipo de salud para desarrollar programas de seguimiento apropiados y estructurados a pacientes con cáncer de mama que requieren intervención quirúrgica.

Es fundamental la implementación de esta intervención educativa de enfermería en los centros de atención oncológica y unidades funcionales de cáncer de mama para lograr una atención integral de mujeres que se enfrentan a esta condición.

\section{Referencias bibliográficas}

1. Izydorczyk B, Kwapniewska A, Lizinczyk S, Sitnik-Warchulska K. Psychological resilience as a protective factor for the body image in post-mastectomy women with breast cancer. Int J Environ Res Public Health 2018; 15:1181. doi: 10.3390/ijerph15061181.

2. Ministerio de Salud y Protección Social. Colombia. Cáncer de mama. 2019. [Acceso el 10 de julio de 2019]. Disponible en: https://www.minsalud.gov.co/salud/publica/ssr/ Paginas/Cancer-de-mama.aspx

3. International Agency for research on cancer. The global cancer observatory. Colombia Source: Globocan 2018. [Acceso el 10 de julio de 2019]. Disponible en: https://gco.iarc. fr/today/data/factsheets/populations/170-colombia-fact-sheets.pdf

4. Sherman KA, Woon S, French J, Elder E. Body image and psychological distress in nipple-sparing mastectomy: the roles of self-compassion and appearance investment. Psychooncology 2017; 26: 337-45. doi: 10.1002/pon.4138.

5. Grogan S, Mechan J. Body image after mastectomy:Athematic analysis of younger women's written accounts. J Health Psychol 2017; 22: 1480-90. doi: 10.1177/1359105316630137.

6. Mushtaq M, Naz F. Body image satisfaction, distress and resilience in women with breast cancer surgery: a within group study. Postgr Med Inst 2017; 31: 39-43.

7. Van De Grift TC, Kreukels BPC, Elfering L, Özer M, Bouman M-B, Buncamper ME, et al. Body image in transmen: multidimensional measurement and the effects of mastectomy. J Sex Med 2016; 13: 1778-86. doi: 10.1016/j.jsxm.2016.09.003.

8. Lilja Y, Rydén S, Fridlund B. Effects of extended preoperative information on perioperative stress: an anaesthetic nurse intervention for patients with breast cancer and total hip replacem. Intensive Crit Care Nurs 1998; 14:276-82. doi: 10.1016/S09643397(98)80688-5

9. Boman LE, Sandelin K, Wengström Y, Silén C. Patients' participation during treatment and care of breast cancer - a possibility and an imperative. Eur J Oncol Nurs 2018; 37:35-42. doi: 10.1016/j.ejon.2018.09.002.

10. Castro EM, Van Regenmortel T, Vanhaecht K, Sermeus W, Van Hecke A. Patient empowerment, patient participation and patient-centeredness in hospital care: A concept analysis based on a literature review. Patient Educ Couns 2016; 99: 1923-39. doi: 10.1016/j.pec.2016.07.026.

11. Eldh AC, Ekman I, Ehnfors M. A comparison of the concept of patient participation and patients' descriptions as related to healthcare definitions. Int J Nurs Terminol Classif 2010; 21:21-32. doi: 10.1111/j.1744-618X.2009.01141.x

12. Waller A, Forshaw K, Bryant J, Carey M, Boyes A, Sanson-Fisher R. Preparatory education for cancer patients undergoing surgery: A systematic review of volume and quality of research output over time. Patient Educ Couns 2015; 98:1540-9. doi: 10.1016/j. pec.2015.05.008 
13. Esteves MT, De Domenico EBL, Petito EL, Gutiérrez MGR de. Intervenção educativa para o automonitoramento da drenagem contínua no pós-operatório de mastectomia. Rev Gaúcha Enferm 2013; 34: 75-83. doi: 10.1590/S1983-14472013000400010

14. Jeffs E, Ream E, Shewbridge A, Cowan-Dickie S, Crawshaw D, Huit M, et al. Exploring patient perception of success and benefit in self-management of breast cancer-related arm lymphoedema. Eur J Oncol Nurs 2016; 20: 173-83. doi: 10.1016/j.ejon.2015.08.001

15. Loh SY, Packer T, Chinna K, Quek KF. Effectiveness of a patient self-management programme for breast cancer as a chronic illness: a non-randomised controlled clinical trial. J Cancer Surviv 2013;7:331-42. doi: 10.1007/s11764-013-0274-x

16. Ahmadidarrehsima S, Rahnama M, Afshari M, Asadi Bidmeshki E. Effectiveness of teach-back self-management training program on happiness of breast cancer patients. Asian Pac J Cancer Prev 2016; 17: 4555-61. doi: 10.22034/APJCP.2016.17.10.4555

17. Smith-Turchyn J, Morgan A, Richardson J. The effectiveness of group-based selfmanagement programmes to improve physical and psychological outcomes in patients with cancer: a systematic review and meta-analysis of randomised controlled trials. Clin Oncol 2016; 28:292-305. doi: 10.1016/j.clon.2015.10.003

18. Carrillo GM, Sanchez B, Vargas Rosero E. Desarrollo y pruebas psicométricas del Instrumento "cuidar"- versión corta para medir la competencia de cuidado en el hogar. Rev Univ Ind Santander Salud [online]. 2016; 48:222-31. doi: 10.18273/revsal.v48n22016007.

19. Consejo de Organizaciones Internacionales de las Ciencias Médicas (CIOMS)/ Organización Mundial de la Salud (OMS). Pautas éticas internacionales para la investigación biomédica en seres humanos. Ginebra: 2002. [Acceso el 4 de julio de 2019]. Disponible en: https:/cioms.ch/wp-content/uploads/2017/12/CIOMS-EthicalGuideline SP_INTERIOR-FINAL.pdf

20. República de Colombia. Ministerio de Salud y Protección Social. Resolución n. ${ }^{\circ} 008430$ de 1993, por la cual se establecen las normas científicas, técnicas y administrativas para la investigación en salud (04/10/1993). [Acceso el 4 de julio de 2019]. Disponible en. https:// www.minsalud.gov.co/sites/rid/Lists/BibliotecaDigital/RIDE/DE/DIJ/RESOLUCION8430-DE-1993.PDF

21. Melo Melo BG, Vargas Hernández Y, Carrillo GM, Alarcón Trujillo DK. Efecto del programa Plan de egreso en la díada persona con enfermedad crónica y sus cuidadores familiares. Enferm Clín 2018; 28: 36-43. doi: 10.1016/j.enfcli.2017.09.006

22. Carrillo GM, González Vega LA. Intervención telefónica y competencia para cuidar de personas con enfermedad crónica. Rev Cienc y Cuid 2018; 15:98-109. 10.22463/17949831.1225

23. Instituto Nacional de Cancerología. Análisis de Situación del Cáncer en Colombia. Bogotá; 2015. [Acceso el 10 de julio de 2019]. Disponible en: http://www.cancer.gov.co/ Situacion_del_Cancer_en_Colombia_2015.pdf

24. Chambers SK, Girgis A, Occhipinti S, Hutchison S, Turner J, McDowell M, et al. A randomized trial comparing two low-intensity psychological interventions for distressed patients with cancer and their caregivers. Oncol Nurs Forum 2014 ; 4: E256-66. doi: 10.1188/14.ONF.E256-E266.

25. Qu B, Luo T. The effects of nursing education on the quality of life in women with breast cancer: A descriptive literature review. Thesis. 2018. Lishui University. [Acceso el 10 de julio de 2019]. Disponible en: http://www.diva-portal.se/smash/get/diva2:1223237/ FULLTEXT01.pdf 
26. Beaver K, Twomey M, Witham G, Foy S, Luker KA. Meeting the information needs of women with breast cancer: Piloting a nurse-led intervention. Eur J Oncol Nurs 2006; 10:378-90. doi: 10.1016/j.ejon.2006.02.004

27. Wagner EH, Ludman EJ, Aiello Bowles EJ, Penfold R, Reid RJ, Rutter CM, et al. Nurse navigators in early cancer care: a randomized, controlled trial. J Clin Oncol 2014; 32:128. doi: 10.1200/JCO.2013.51.7359

28. Gilbert JE, Green E, Lankshear S, Hughes E, Burkoski V, Sawka C. Nurses as patient navigators in cancer diagnosis: review, consultation and model design. Eur J Cancer Care. 2011; 20:228-36. doi: 10.1111/j.1365-2354.2010.01231.x

29. Ludman EJ, McCorkle R, Bowles EA, Rutter CM, Chubak J, Tuzzio L, et al. Do depressed newly diagnosed cancer patients differentially benefit from nurse navigation? Gen Hosp Psychiatry 2015; 37:236-9. doi: 10.1016/j.genhosppsych.2015.02.008 